\title{
Evaluation of aortic regurgitation with cardiac magnetic resonance imaging: a systematic review
}

\author{
James C Lee, ${ }^{1}$ Kelley R Branch, ${ }^{1}$ Christian Hamilton-Craig, ${ }^{1,2,3}$ Eric V Krieger ${ }^{1,4}$
}

\begin{abstract}
${ }^{1}$ Division of Cardiology, Department of Medicine, University of Washington, Seattle, Washington, USA ${ }^{2}$ Centre for Advanced Imaging, University of Queensland, Brisbane, Queensland, Australia ${ }^{3}$ Department of Cardiology, Heart \& Lung Institute, The Prince Charles Hospital, Brisbane, Queensland, Australia ${ }^{4}$ Seattle Adult Congenital Heart Service, University of Washington School of Medicine, Seattle, Washington, USA
\end{abstract}

\section{Correspondence to}

Dr Eric V Krieger, Seattle Adult Congenital Heart Service,

University of Washington School of Medicine, Seattle, WA 98195 USA; eKrieger@cardiology. washington.edu

Revised 31 July 2017 Published Online First 19 August 2017

\section{CrossMark}

To cite: Lee JC, Branch KR, Hamilton-Craig C, et al. Heart 2018;104:103-110.

\section{ABSTRACT}

This review summaries the utility, application and data supporting use of cardiac magnetic resonance imaging (CMR) to evaluate and quantitate aortic regurgitation. We systematically searched Medline and PubMed for original research articles published since 2000 that provided data on the quantitation of aortic regurgitation by CMR and identified 11 articles for review. Direct aortic measurements using phase contrast allow quantitation of volumetric flow across the aortic valve and are highly reproducible and accurate compared with echocardiography. However, this technique requires diligence in prescribing the correct imaging planes in the aorta. Volumetric analytic techniques using differences in ventricular volumes are also highly accurate but less than phase contrast techniques and only accurate when concomitant valvular disease is absent. Comparison of both aortic and ventricular data for internal data verification ensures fidelity of aortic regurgitant data. CMR data can be applied to many types of aortic valve regurgitation including combined aortic stenosis with regurgitation, congenital valve diseases and post-transcatheter valve placement. CMR also predicts those patients who progress to surgery with high overall sensitivity and specificity. Future studies of CMR in patients with aortic regurgitation to quantify the incremental benefit over echocardiography as well as prediction of cardiovascular events are warranted.

\section{INTRODUCTION}

Chronic aortic regurgitation (AR) is a state of increased left ventricular (LV) volume causing elevated preload and afterload, which, if untreated, causes LV dilation and dysfunction. ${ }^{1}$ Echocardiography remains the mainstay for the evaluation of $\mathrm{AR}$ as it is widely available, can measure ventricular size and function and estimates the severity of AR. However, cardiac magnetic resonance (CMR) has superior reproducibility for ventricular volumes and systolic function measurements, ${ }^{2}$ can quantitate AR severity with precision not possible by echocardiography, and can identify ventricular fibrosis-a consequence of long-standing AR. This article reviews the different CMR techniques for AR evaluation and compares CMR measures with echocardiography. We discuss whether the improved precision provided by CMR results in improved risk stratification or patient outcomes. Finally, we discuss the use of CMR to evaluate paravalvular AR in patients who have undergone a transcatheter aortic valve replacement (TAVR).

\section{METHODS}

\section{Systematic review}

A systematic review was performed in accordance with the 2015 Preferred Reporting Items for Systematic Reviews and Meta-Analysis methodology. ${ }^{3}$ PubMed and Medline were queried for studies published in English since 2000. Search terms of 'aortic regurgitation' or 'aortic insufficiency' AND 'magnetic resonance' identified 52 articles. Two reviewers (EVK and JCL) selected articles with adjudication performed by consensus. Exclusion criteria were as follows: (1) studies that used qualitative data without evaluating quantitative CMR techniques, (2) review articles, editorials or abstracts, (3) studies that did not use modern imaging sequences such as steady state free progression (SSFP) imaging and (4) studies with fewer than 10 subjects or non-human subjects. Sections on reproducibility included studies that reported intraobserver and interobserver reproducibility. Comparison with echocardiography included studies that described a quantitative AR cut-off for CMR. Eleven studies (21\%) met inclusion criteria and were included in this systematic review.

\section{Aortic regurgitation assessment}

Many imaging tools currently exist for the evaluation of AR (table 1). However, echocardiography remains the primary imaging modality for initial evaluation and longitudinal assessment of AR. Echocardiography uses an integrative approach to evaluate valve morphology, estimate regurgitation severity and assess ventricular response to chronic volume overload. ${ }^{4-7}$ While qualitative and semi-quantitative measures are reliable markers of AR severity, echocardiography is less well suited for the quantitation of AR volumes (figure 1).

For most patients, echocardiography is sufficient to identify severe AR and CMR is not required. CMR adds the most value for patients with suboptimal echocardiography, indeterminate AR severity, following TAVR or inconclusive Doppler data, for which CMR was given a class I recommendation (level of evidence B) in the 2014 American Heart Association/American College of Cardiology guidelines. $^{6}$

\section{How to quantify aortic regurgitation using CMR} CMR uses a combination of ventricular volume and aortic flow data to assess AR severity quantitatively. Myocardial function is evaluated with bright-blood cine images that have excellent delineation between blood and myocardium without the 
Table 1 Multimodality tools to evaluate for aortic regurgitation

\begin{tabular}{|c|c|c|c|c|c|}
\hline & $\begin{array}{l}\text { LV size/function/wall motion/ } \\
\text { mass }\end{array}$ & $\begin{array}{l}\text { Aortic valve } \\
\text { morphology }\end{array}$ & $\begin{array}{l}\text { Aortic stenosis } \\
\text { quantitation }\end{array}$ & $\begin{array}{l}\text { Aortic regurgitant } \\
\text { volume quantitation }\end{array}$ & $\begin{array}{l}\text { Paravalvular regurgitation } \\
\text { quantitation }\end{array}$ \\
\hline TTE & ++ & ++ & +++ & + & + \\
\hline TOE & + & +++ & ++ & + & ++ \\
\hline CMR & +++ & +++ & + & +++ & +++ \\
\hline MDCT & ++ & ++ & - & - & - \\
\hline Invasive angiography & ++ & - & +++ & & ++ \\
\hline
\end{tabular}

Many tools now exist for the evaluation of valvular heart disease. This table shows the relative utility of each of these tools for the interrogation of specific issues related to aortic valve disease

CMR, cardiovascular magnetic resonance imaging; LV, left ventricle; MDCT, multidetector CT; TOE, transoesophageal echocardiography; TTE, transthoracic echocardiography.

need for intravascular contrast. Cine images also show valve morphology and pathology such as leaflet prolapse. Tracing the endocardial and epicardial borders on a stack of short axis cine images provides highly reproducible LV volumes, mass and ejection fraction. The reproducibility of LV volumetric analysis makes CMR effective in evaluating and tracking ventricular size and function both before and after valvular intervention. ${ }^{8}$ Advantages of CMR include lack of ionising radiation and no limitations in the number or orientation of imaging planes. CMR is limited by arrhythmia, artefacts, longer scan time and patient tolerance.

\section{Direct aortic flow measurements}

The most commonly used method to quantify AR is direct measurement using phase contrast (PC) imaging. PC CMR is analogous to Doppler echocardiography and measures flow velocity and direction over time. PC CMR imaging is typically performed through an imaging plane ('though-plane') in the ascending aorta for the measurement of AR. Blood flow is measured into and out of the aortic plane over time to generate flow curves. Integration of the flow curves allows the calculation of stroke volume (total forward flow), cardiac output (stroke volume $\mathrm{x}$ heart rate), regurgitant volume (total backward flow) and the regurgitant fraction (regurgitant volume/stroke volume).
Similar to Doppler, PC CMR images can also measure the velocity of blood flow through the valve up to the maximum velocity-encoding (VENC) limit. This is useful in patients with combined AR and stenosis (figure 2).

\section{Advantages}

Direct PC CMR measurement of aortic forward and retrograde flow requires only a single breath hold and the post-processing time is minimal. Phase velocity mapping is highly reproducible and accurate, ${ }^{9-11}$ and the predominant sources of variation occurs at the time of image acquisition (table 2). ${ }^{1012-14}$

\section{Potential pitfalls}

PC CMR technique assumes that blood flow is laminar and the imaging planes are aligned perpendicular to blood flow in a double oblique orientation. In patients with abnormal aortic valves, these assumptions are often untrue. Furthermore, slice location affects the aortic flow measurements: as the slice plane moves distally from the aortic valve, regurgitant fraction progressively decreases. ${ }^{15} 16$ Moving the slice location between the aortic sinuses, the sinotubular junction and the mid-ascending aorta can change forward stroke volume by up to $15 \%$ and regurgitant volume measurements by up to $20 \%$. $^{15} 17$ Variations
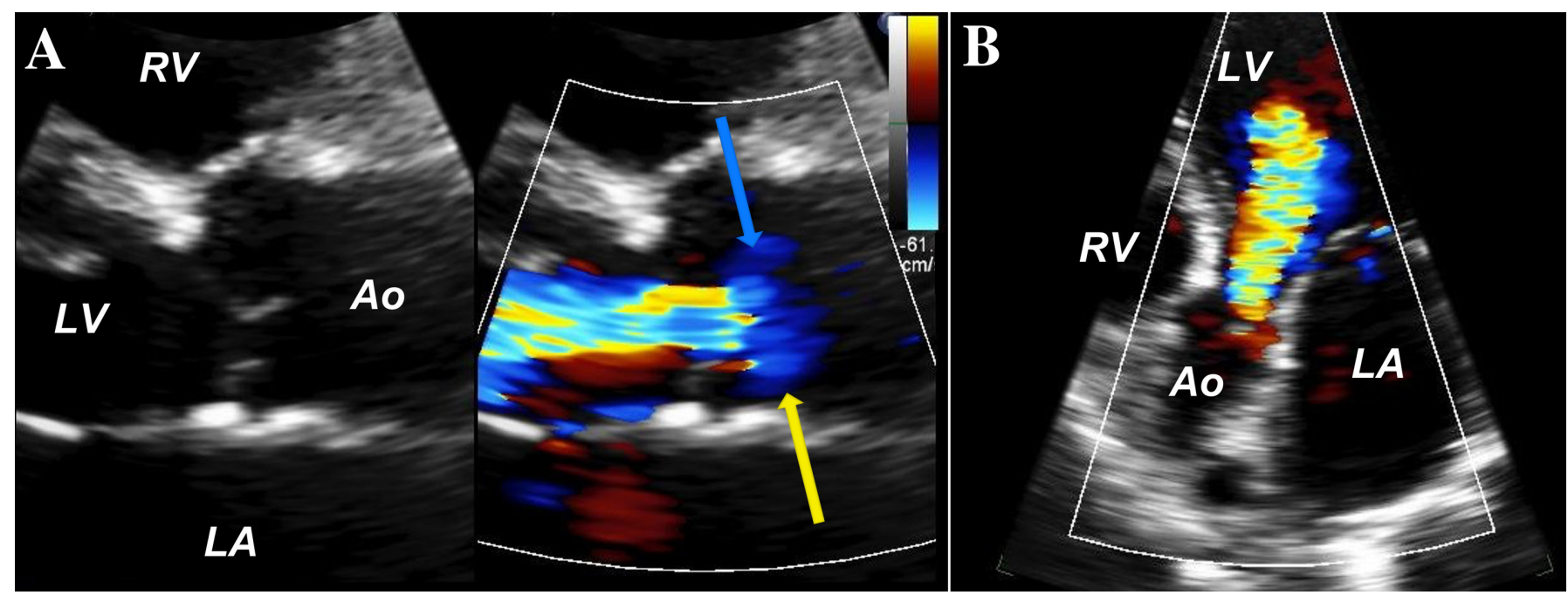

Figure 1 Limitations of proximal isovelocity surface area (PISA) technique for quantitation of aortic regurgitation with echocardiography. (A) Sideby-side comparison of aortic valve with colour Doppler comparison. Blue arrow shows the typical of incidence of Doppler to the regurgitant jet in the parasternal axis from transthoracic echocardiogram (TTE) and the yellow arrows shows the typical angle of incidence of Doppler to the regurgitant jet from transoesophageal echocardiogram. Unless there is a very eccentric jet of aortic regurgitation, this will not be conducive to use the PISA technique to quantitate aortic regurgitation. (B) Using TTE apical windows, with good image quality, the more parallel alignment of flow with is in theory more ideal for measuring a PISA radius. However, as the PISA envelope will be in the far field, reductions in temporal and spatial resolution again limit accurate measurements. Ao, aortic; LA, left atrium; LV, left ventricle; RV, right ventricle. 


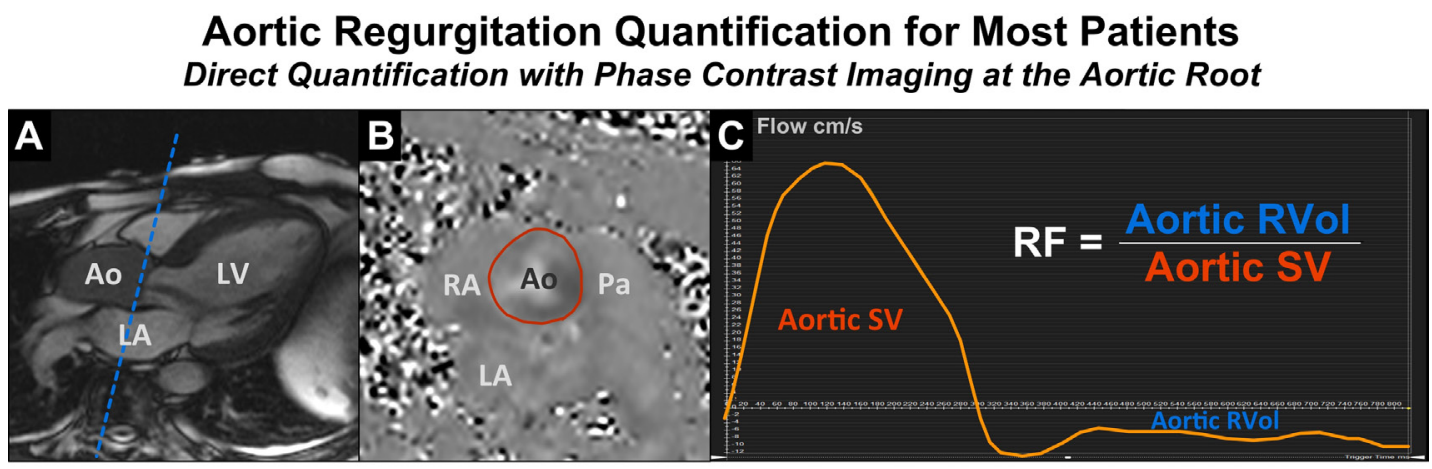

\section{Concern for Accuracy of Direct Quantification of Regurgitant Volume Comparison of Aortic Forward Flow and Pulmonary Forward Flow}

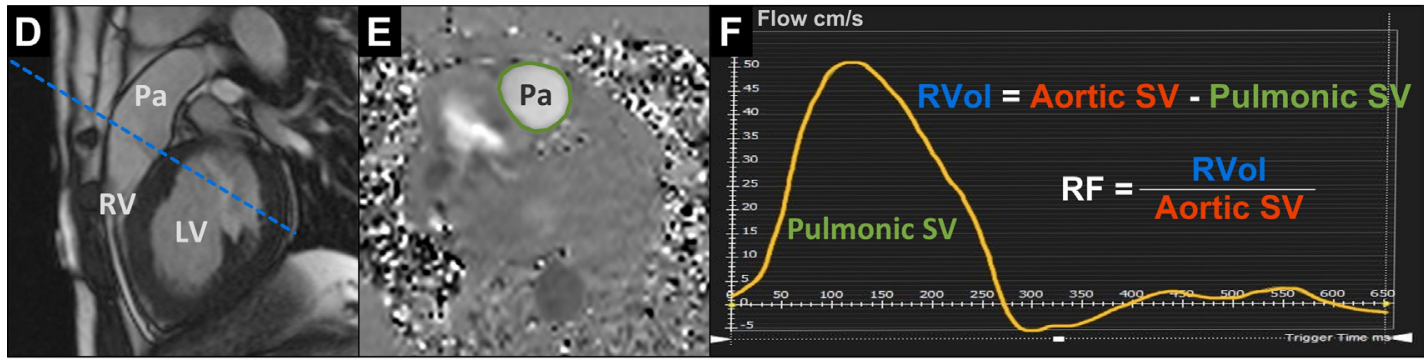

\section{Concern for Accuracy of Entire Aortic Phase Contrast Image Comparison of Right and Left Ventricular Stroke Volumes}

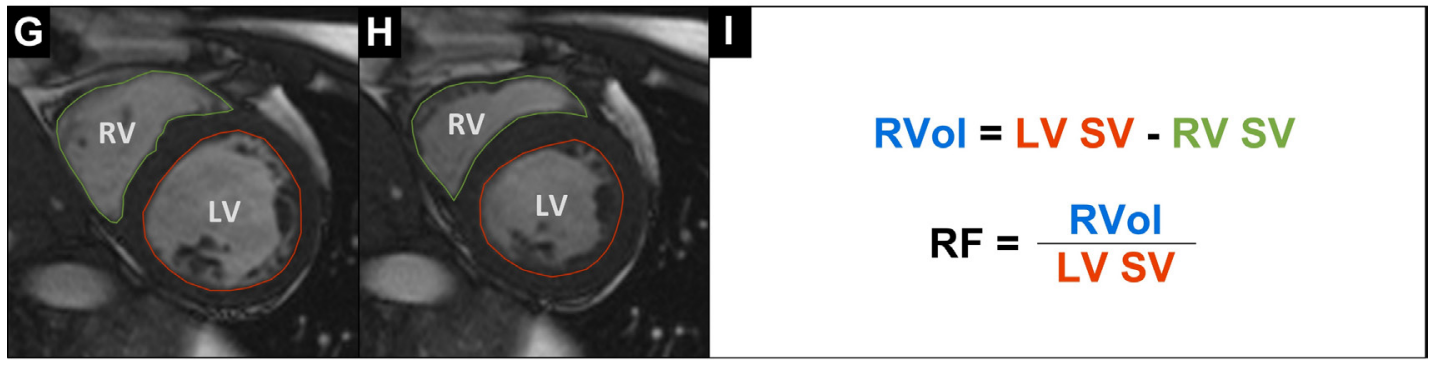

Figure 2 Cardiovascular magentic resonance imaging for quantitation of aortic regurgitation. (A) Blue dotted line shows one of two 90 degree double oblique planes prescribed to obtain a phase contrast $(P C)=$ flow sequence at the aortic valve. (B) PC flow sequence at the cross-section of the aortic valve with its area traced in red by manual planimetry. (C) Using semi-automated software, forward and regurgitant flows are directly quantitated from PC flow sequences and used to derive a regurgitant volume and regurgitant fraction. If there is concern regarding the accuracy of the aortic regurgitant flow, an alternative methodcan be used which uses the difference between forward aortic and pulmonic flows to indirectly calculate an aortic regurgitant volume and aortic regurgitant fraction. (D) Blue dotted line shows one of two 90 degree double oblique planes prescribed to obtain a PC flow sequence at the cross-section of the pulmonic valve. (E) The green circle shows the use of manual planimetry, which when performed throughout the cardiac cycle can be used to obtain the pulmonary forward flow. (F) Flow curve of the pulmonary artery and the formula for derivation of the aortic and pulmonary flow method. (G) If for technical reasons no component of the aortic PC flow is felt to be accurate, the difference in left and right ventricular stroke volumes can also be used to indirectly estimated aortic regurgitant volume and fraction. These volumes can be obtained by tracingthe endocardium on a stack of images of each ventricle and mulitplying by the slice thickness. Performing this over diastole $(\mathrm{G})$ and systole $(\mathrm{H})$ allows for derivation of ventricular volumes. The formula for this is shown in (I). This technique is only valid if there is isolated aortic regurgitation. Ao, aortic; LA, left atrium; LV, left ventricle; Pa, pulmonic; RA, right atrium; RF, regurgitant fraction; RV, right ventricle; RVol, regurgitant volume; SV, stroke volume.

are secondary to coronary blood flow and non-laminar flow patterns from local eddies which do not pass perpendicularly through the plane of acquisition. With turbulent or non-laminar flow at the level of the aortic sinuses, moving the slice location distally, to where the flow is more laminar, is desirable. Despite these potential confounders, regurgitation fraction in practice rarely varies by $>10 \%$ based on slice location.

Similar to Doppler, PC CMR images are dependent on the angle of intercept and small angular deviations underestimate velocity and therefore underestimate flow. ${ }^{18}$ Often, forward flow and retrograde flow do not travel along the same plane so one flow is misaligned and therefore under measured.

Much like the Nyquist limit for Doppler, PC CMR images also have a maximum VENC limit, which, if exceeded, results in aliasing of the signal and underestimation of peak velocity. When aliasing occurs (which is common with higher velocities seen in aortic stenosis, after aortic valve replacement or in high flow states), the VENC limit should be changed to a higher value 
Table 2 Cardiovascular magnetic resonance imaging and aortic regurgitation reproducibility

\begin{tabular}{|c|c|c|c|c|c|c|}
\hline First author/year & $\begin{array}{l}\text { Number of patients } \\
\text { quantitatively } \\
\text { analysed }(n=)\end{array}$ & Population & Study design & $\begin{array}{l}\text { Quantitative CMR } \\
\text { methodology }\end{array}$ & $\begin{array}{l}\text { Intraobserver } \\
\text { variability }\end{array}$ & $\begin{array}{l}\text { Interobserver } \\
\text { variability }\end{array}$ \\
\hline Salaun et al/2016 & 26 & Post-TAVR & $\begin{array}{l}\text { Prospective single } \\
\text { centre }\end{array}$ & $\begin{array}{l}\text { Direct flow: CMR } \\
\text { flow assessed at four } \\
\text { different levels }\end{array}$ & ICC: $0.99(0.97-1)$ & ICC: $0.99(0.97-0.99)$ \\
\hline Frick et al/2016 & 69 & Post-TAVR & $\begin{array}{l}\text { Prospective single } \\
\text { centre }\end{array}$ & $\begin{array}{l}\text { Direct flow: slice place } \\
\text { just superior to aortic } \\
\text { valve prosthesis }\end{array}$ & $\begin{array}{l}\text { RVOL: } 2.2 \pm 1.9 \mathrm{~mL} \\
\text { (ICC: } 0.999)\end{array}$ & $\begin{array}{l}\text { RF: } 1.5 \% \pm 1.1 \% \\
\text { (ICC: } 0.999)\end{array}$ \\
\hline Altiok et al/2014 & 71 & Post-TAVR & $\begin{array}{l}\text { Prospective single } \\
\text { centre }\end{array}$ & $\begin{array}{l}\text { Direct flow: slice just } \\
\text { superior to valve } \\
\text { prosthesis }\end{array}$ & $\begin{array}{l}\text { RVOL: } 2.2 \% \pm 2.0 \% \\
\text { RF: } 1.9 \% \pm 1.9 \%\end{array}$ & $\begin{array}{l}\text { RVOL: } 1.5 \% \pm 1.5 \% \\
\text { RF: } 1.7 \% \pm 1.1 \%\end{array}$ \\
\hline Cawley et al/2013 & 31 & $\begin{array}{l}\text { Chronic aortic } \\
\text { regurgitation }\end{array}$ & $\begin{array}{l}\text { Prospective single } \\
\text { centre }\end{array}$ & $\begin{array}{l}\text { Direct flow: slice } \\
\text { located at the level of } \\
\text { the aortic sinuses }\end{array}$ & $\begin{array}{l}\text { RVOL: } 0 \text { ( }-2 \text { to } 2), \\
r=0.99 \\
\text { RF: } 0 \text { (-2 to } 2), r=0.99\end{array}$ & $\begin{array}{l}\text { RVOL: }-0.7 \text { ( }-5 \text { to } 4) \text {, } \\
r=0.99 \\
\text { RF: }-0.7 \text { ( }-4 \text { to } 3) \text {, } \\
r=0.99\end{array}$ \\
\hline
\end{tabular}

The reproducibility of CMR for the quantification of aortic regurgitation for both native valvular heart disease as well as after transcatheter aortic valve replacement is excellent. This is true despite a heterogeneity of sampling locations used in the aortic root.

CMR, cardiovascular magnetic resonance imaging; ICC, intraclass correlation coefficient; RF, regurgitant fraction; RVOL, regurgitant volume; TAVR, transcatheter aortic valve replacement.

and the acquisition repeated. Because PC CMR has relatively low temporal resolution as compared with echocardiography, the peak systolic velocity may be underestimated, ${ }^{19}$ and results in underestimation of forward flow volume, particularly in patients with concomitant aortic stenosis. ${ }^{112021}$ Finally, the slice thickness for the velocity encoded flow technique leads to partial volume effects, which will also systematically underestimate the peak velocity and therefore the peak flow. With high VENC, direct $\mathrm{AR}$ quantitation is somewhat less accurate, snd when a high VENC is needed ( $>\sim 3 \mathrm{~m} / \mathrm{s})$, confirmation of AR volume using a secondary technique is advisable.

Because PC CMR data are obtained in segments over several heartbeats, an irregular rhythm (such as frequent premature ventricular contractions or atrial fibrillation) reduces the accuracy of flow measurements. Most vendors employ arrhythmia rejection algorithms, which exclude beats with very divergent $\mathrm{R}-\mathrm{R}$ intervals, but rejecting too many beats significantly increases scan time making breath-holding difficult. Thus, in patients with significant heart rate variability, flow data and volumetric data are often imprecise. Arrhythmia affects the other CMR techniques discussed below as well and quantification is often unreliable in these patients. Advances in real-time single-beat acquisition may overcome these limitations in the future. ${ }^{22}$

\section{Comparison of forward aortic flow and pulmonary forward flow}

AR can be calculated by subtracting the net flow through the pulmonary artery from the total flow through the aorta. This relies on the principle that, in the absence of a shunt or regurgitation, the net volume of blood leaving the left ventricle into the aorta and right ventricle into the pulmonary artery are equal. In $\mathrm{AR}$, the total aortic blood flow will be higher than the pulmonic flow and the difference is the AR regurgitant volume.

\section{Advantages}

This method is most commonly used if there is concern about the accuracy of the direct quantitation of regurgitant volume and can also serve as an internal validation. This technique is useful in patients with aortic stenosis where the higher VENC leads to underestimation of regurgitant volume. Some groups have questioned the reliability of the direct PC CMR measurements of aortic regurgitant volumes because AR volume varies depending on the slice location in the aorta, as discussed above, and this technique is appealing in that it does not rely on direct measurement of small AR volume. ${ }^{151723}$

\section{Potential pitfalls}

It can be difficult to obtain a perfect double oblique cross-sectional plane through the proximal main pulmonary artery due to its curvature. This technique is also inaccurate in patients with shunts. Additionally, if heart rate or haemodynamics change between the acquisition of the flow through the aorta and the pulmonary artery, results are inaccurate.

\section{Variations and internal validation}

A strength of CMR is the availability of multiple techniques, which can provide internal validation for AR measures. We do not recommend that every technique described be used in all cases, but these techniques are useful to address discrepancies or as backup modalities if technical issues with PC CMR image acquisition occur (figure 2).

- In patients with isolated AR (no other valvular abnormalities or shunt), the difference between the right ventricular and LV stroke volumes is the regurgitant volume. However, the reproducibility of right ventricular stroke volumes is lower than LV stroke volume so the reproducibility of this technique is typically lower than the reproducibility of PC CMR.

- In patients with coexisting AS (which can lead to underestimation of aortic forward flow), AR volume can be calculated by subtracting the net flow through the pulmonary artery from the LV stroke volume. This method is inaccurate if there is mitral regurgitation.

\section{Other complementary CMR techniques for aortic regurgitation}

Beyond direct quantitation, CMR offers other ways to evaluate the aortic valve in AR. For example, some centres have shown correlation between planimetry of the regurgitant orifice area CMR-derived regurgitant fraction and regurgitant volume $(r=0.9, p<0.001) .{ }^{24}$ Holodiastolic flow reversal in the descending aorta with PC CMR supports the diagnosis of severe $\mathrm{AR}^{25}$ 


\section{DOES CMR OF AORTIC REGURGITATION AID IN CLINICAL MANAGEMENT?}

Because CMR is accurate and reproducible for the assessment of AR, it seems well suited to guide management by providing prognostic information to inform timing for aortic valve replacement. However, there has been relatively little published data on the additive clinical value of CMR; most literature addresses questions of reproducibility and accuracy, rather than patient outcomes. The available studies are small, vulnerable to biases, but the results are promising (table 3). ${ }^{26-28}$ Myerson et al reported on 113 patients with at least moderate chronic AR. Over 9 years of follow-up, 35\% underwent aortic valve replacement for symptoms, progressive aortic dilation or LV systolic dysfunction. A regurgitant fraction $>33 \%$ had $85 \%$ sensitivity and $92 \%$ specificity for identifying patients who would progress to surgery. No patients with a regurgitation fraction $<26 \%$ progressed to surgery. As discussed below, these values are considerably lower than the cut-off for severe AR used in echocardiography. Severe LV dilation (LVEDV $>246 \mathrm{~mL}$ ) was also independently associated with the need for surgery in follow-up. Harris et al reported that CMR was superior to echocardiography in predicting which patients would require surgery for chronic AR. Twenty-nine asymptomatic patients who underwent transthoracic echocardiography (TTE) and CMR on the same day were followed for 4 years. CMR was superior to TTE at identifying the patients who required an aortic valve replacement. A regurgitant fraction of $>37 \%$ had a sensitivity of $100 \%$ and specificity of $75 \%$ for requiring valve surgery during follow-up. ${ }^{26}$

There is centre-to-centre variability in regard to which regurgitant volume and fraction cut-offs define severe AR. The papers by Myerson et al and Harris et al suggest that outcomes are worse for patients with $>33 \%$ AR. In our labs, we define mild-AR as $<20 \%$, moderate AR as $20 \%-40 \%$ and severe AR $\geq 40 \%$. It is important to note that these cut-offs are largely arbitrary designations with the limited data to validate their clinical significance. Refinement of these data thresholds are an important topic for future research. ${ }^{29}$

\section{COMPARISON OF AORTIC REGURGITATION MEASURED BY ECHOCARDIOGRAPHY AND CMR}

Because echocardiography primarily uses an integrative semi-quantitative method and CMR uses a quantitative approach to evaluate AR, directly comparing the two techniques is difficult. CMR can reproduce many of the parameters used in the TTE integrative approach, but these findings are rarely prioritised over the quantitative approach. For example, flow reversal of the descending aorta using through-plane PC CMR correlates well with an echocardiographic diagnosis of severe AR. ${ }^{25}$ Unlike the width of the colour Doppler signalwhich is an accepted echocardiographic measure of AR severity - the size of the AR dephasing jet seen on CMR is unreliable and is dependent on multiple factors including flip angle and echo time. ${ }^{30}$

Echocardiography can quantify AR volume and regurgitant fraction using either the PISA technique or calculated right ventricular and LV stroke volumes based on Doppler outflow measures. AR volume measured by echocardiography tend to be higher than CMR-derived regurgitation volume in native valve regurgitation and lower than CMR following TAVR. Overall correlation is modest. ${ }^{102429}$

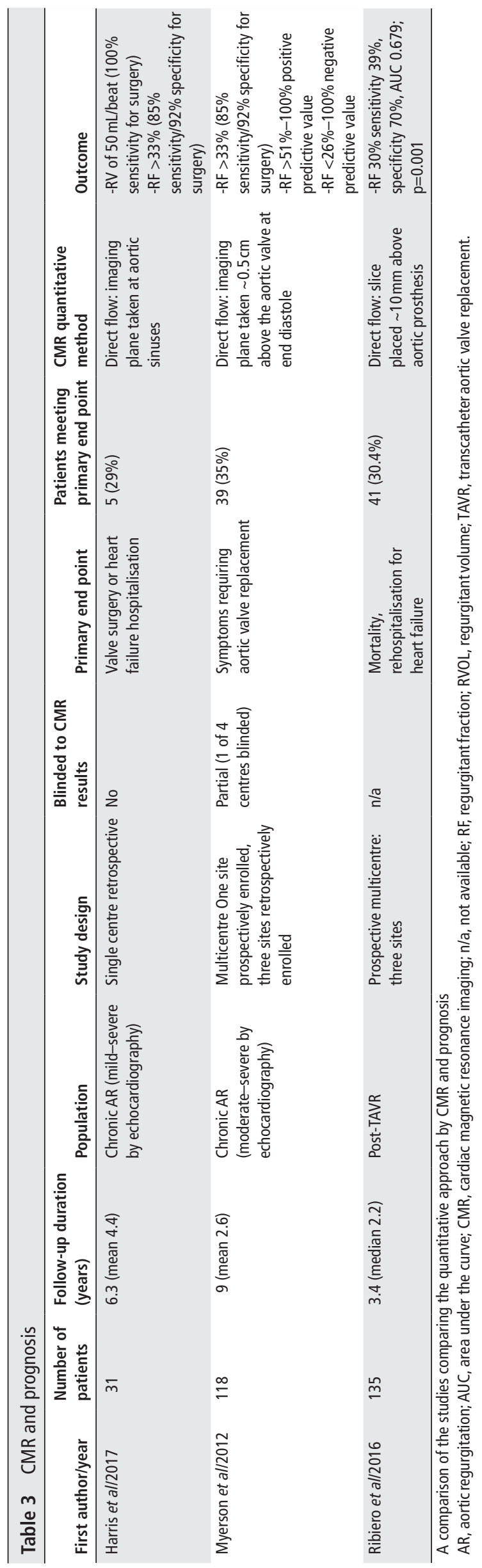




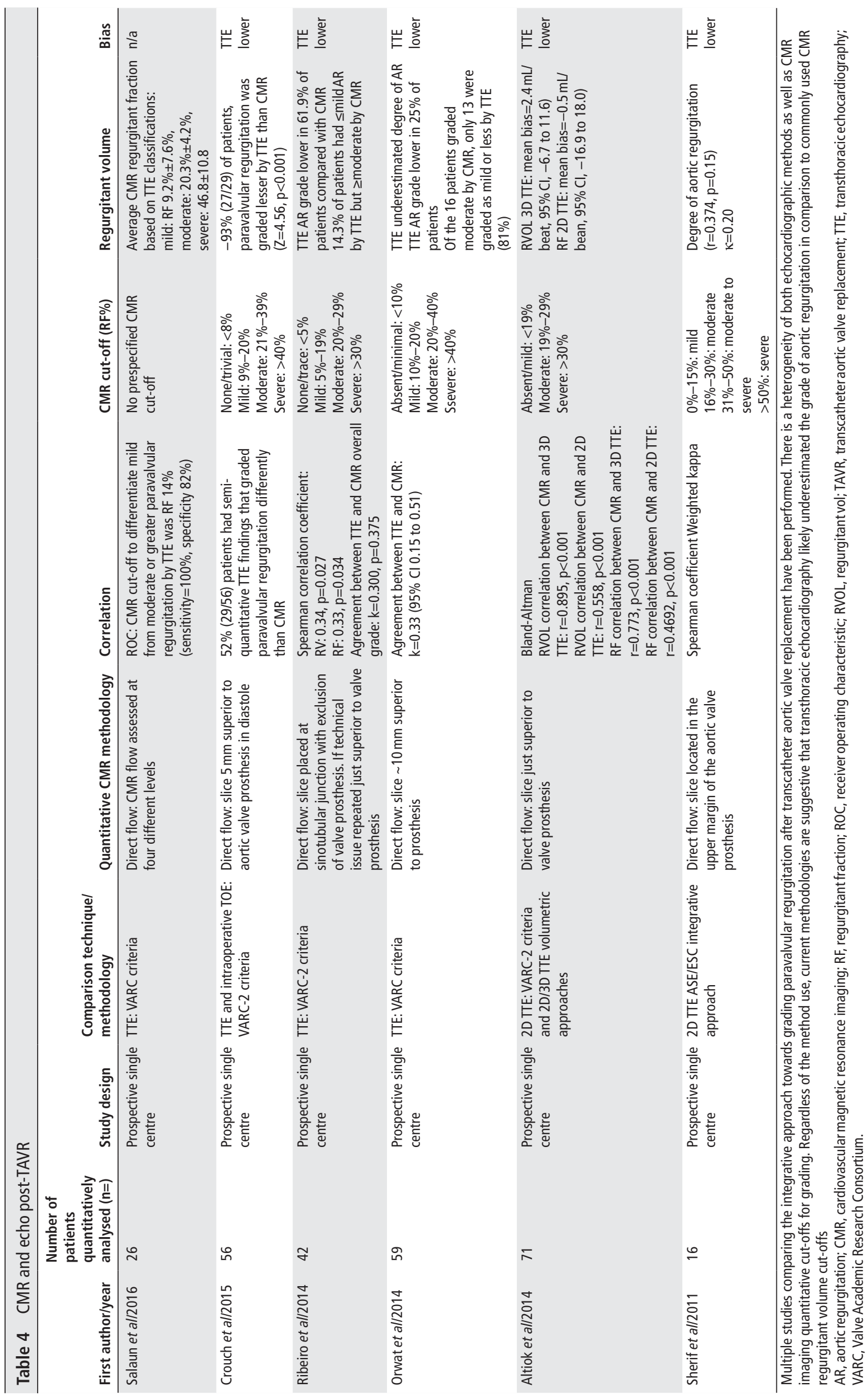




\section{OTHER APPLICATIONS OF CMR IN AORTIC REGURGITATION EVALUATION \\ Cardiac magnetic resonance in TAVR \\ Pre-TAVR planning}

CMR can be used for preprocedural planning of TAVR, although multidetector computed tomography is used more commonly. $\mathrm{CMR}$ is most valuable in patients with tenuous renal function or severe allergies to iodinated contrast. With respiratory-navigated ECG gated isotropic sequences, CMR can obtain aortic annulus size, shape and dynamic annular motion, but require long scan times. Vascular access planning can be completed with gadolinium magnetic resonance angiography.

\section{Post-TAVR evaluation}

Following TAVR, the presence of a paravalvular leak (PVL) is associated with worse clinical outcomes so the diagnosis and quantitation of PVL is an important goal of follow-up imaging. ${ }^{31-33}$ While TTE has a high sensitivity for detecting even small PVL, echocardiographic grading of the severity is difficult because AR jets are commonly eccentric, crescentic and shadowed by the valve stent. ${ }^{34}$ It is rare to quantify PVL regurgitant volume or fraction successfully by echocardiography and the standard integrative echocardiographic approach for native AR is not validated following TAVR. Most experts suggest using the per cent circumference involved in the short-axis view to grade the severity of PVL, where $>30 \%$ of the valve circumference is considered severe PVL according to the Valve Academic Research Consortium-2 consensus statement. ${ }^{35}$ This approach has several shortcomings including poor interobserver reproducibility and lack of validation of this cut-off against clinical end points. TOE sometimes provides better views of PVL but requires sedation, does not allow for reliable quantitation and cannot overcome the difficulty in assessing multiple eccentric crescentic jets.

Compared with echocardiography, CMR has improved reproducibility in quantifying PVL following TAVR. ${ }^{14}$ However, due to the metallic stent that holds the valve, slice location needs to be planned carefully. The best location for PC CMR is never at the level of the valve due to susceptibility artefact from the stent. In patients with a Sapien valve (Edwards Lifescience, Irvine, California, USA), the optimal slice location appears to be at the sinotubular junction. Patients who have a CoreValve (Medtronic, Minneapolis, Minnesota, USA) should have the slice location placed higher, at the tubular portion of the ascending aorta. $^{12}$

Several studies have compared CMR and echocardiography in the grading of PVL following TAVR (table 4). ${ }^{121436-39}$ In general, echocardiography underestimates PVL severity compared with CMR, and patients who are diagnosed with mild PVL by echocardiography often have regurgitation fractions $>20 \%$. $^{12} 142836-39$ This is in contrast to native valve AR when echocardiography overestimates AR severity compared with CMR. While there is no gold standard for PVL assessment, there are theoretical reasons to believe that CMR is more accurate than echocardiography in defining PVL severity. One study showed that CMR performed 40 days after TAVR had stronger association with clinical events than echocardiography. A CMR-derived regurgitation fraction following TAVR also had the strongest correlation with mortality or heart failure hospitalisation. ${ }^{28}$

In patients with more than mild PVL seen on echo, CMR may also identify patients who could benefit from PVL closure. The amount of PVL which warrants closure is unclear but a CMR-derived RF of $>30 \%$ identified patients at risk for 2-year mortality with a sensitivity of $39 \%$ and a specificity of $70 \%$ (area under the curve $0.678, \mathrm{p}=0.001) .^{28}$ It remains uncertain whether occlusion of the PVL reduces that risk.

\section{FUTURE DIRECTIONS}

The development for CMR pulse sequences continues to improve. Acquisition times are improving and may allow for real-time flow acquisitions, which shorten scan time as well as overcome some of the challenges in patients with arrhythmia. ${ }^{22}$ Four-dimensional (4D) flow sequences acquire a large stack of flow data in all three dimensions, and then postprocessing adds flow lines to MRA datasets. The 4D flow accounts for differences in flow patterns and may be more reproducible and accurate for non-laminar flow. ${ }^{40-42}$

Some groups have used CMR data to model wall stress and these types of analysis may ultimately yield novel insights into the pathophysiology of AR. ${ }^{43}$ The use of CMR can be also used to evaluate dynamic changes in peripheral vasculature such as aortic compliance, and may yield insights into some of the heterogeneity of the presentation of $\mathrm{AR}$ and why there can be discrepancies with the echocardiographic integrative approach. ${ }^{44}$

\section{CONCLUSIONS}

CMR provides highly reproducible quantification of $A R$, and the multiple data inputs allow for internal validation to ensure fidelity of AR measures. However, CMR pitfalls exist and knowledge of these are critical to ensure the highest quality data. Note that not all patients with AR require CMR and echocardiography alone can guide care in many patients. However, in patients where echocardiography is inconclusive or discordant with clinical assessment, CMR is an important complementary technique.

Contributors JCL, KRB, CH-C, EVK contributed to the drafting of the study and critical review.

Competing interests $\mathrm{Dr} \mathrm{CH}-\mathrm{C}$ has been an speaker for Siemens, Merck and Edwards. Dr KRB has received research support from Bayer, Astellas. Ad board and Jansen. Drs JCL and EVK have no conflicts to report.

Provenance and peer review Commissioned; externally peer reviewed. Data sharing statement None declared.

(c) Article author(s) (or their employer(s) unless otherwise stated in the text of the article) 2018. All rights reserved. No commercial use is permitted unless otherwise expressly granted.

\section{REFERENCES}

1 Starling MR, Kirsh MM, Montgomery DG, et al. Mechanisms for left ventricular systolic dysfunction in aortic regurgitation: importance for predicting the functional response to aortic valve replacement. J Am Coll Cardiol 1991;17:887-97.

2 Grothues F, Smith GC, Moon JC, et al. Comparison of interstudy reproducibility of cardiovascular magnetic resonance with two-dimensional echocardiography in normal subjects and in patients with heart failure or left ventricular hypertrophy. Am J Cardiol 2002:90:29-34.

3 Moher D, Shamseer L, Clarke M, et al. Preferred reporting items for systematic review and meta-analysis protocols (PRISMA-P) 2015 statement. Syst Rev 2015;4:1.

4 Zoghbi WA, Adams D, Bonow RO, et al. Recommendations for Noninvasive Evaluation of Native Valvular Regurgitation: A Report from the American Society of Echocardiography Developed in Collaboration with the Society for Cardiovascular Magnetic Resonance. J Am Soc Echocardiogr 2017;30.

5 Nishimura RA, Otto CM, Bonow RO, et al. 2017 AHA/ACC Focused Update of the 2014 AHA/ACC Guideline for the Management of Patients With Valvular Heart Disease: A Report of the American College of Cardiology/American Heart Association Task Force on Clinical Practice Guidelines. Circulation 2017;135:e1159-e1195.

6 Nishimura RA, Otto CM, Bonow RO, et al. AHA/ACC Guideline for the Management of Patients With Valvular Heart Disease. A Report of the American College of Cardiology/ American Heart Association Task Force on Practice Guidelines 2014.

7 Lancellotti P, Tribouilloy C, Hagendorff A, et al. Recommendations for the echocardiographic assessment of native valvular regurgitation: an executive summary from the European Association of Cardiovascular Imaging. Eur Heart J CardiovasC Imaging 2013;14:611-44. 
8 Lamb HJ, Beyerbacht HP, de Roos A, et al. Left ventricular remodeling early after aortic valve replacement: differential effects on diastolic function in aortic valve stenosis and aortic regurgitation. J Am Coll Cardiol 2002;40:2182-8.

9 Chatzimavroudis GP, Oshinski JN, Franch $\mathrm{RH}$, et al. Evaluation of the precision of magnetic resonance phase velocity mapping for blood flow measurements. J Cardiovasc Magn Reson 2001;3:11-19.

10 Cawley PJ, Hamilton-Craig C, Owens DS, et al. Prospective comparison of valve regurgitation quantitation by cardiac magnetic resonance imaging and transthoracic echocardiography. Circ Cardiovasc Imaging 2013;6:48-57.

11 Lotz J, Döker $R$, Noeske $R$, et al. In vitro validation of phase-contrast flow measurements at $3 \mathrm{~T}$ in comparison to 1.5 T: precision, accuracy, and signal-to-noise ratios. J Magn Reson Imaging 2005;21:604-10.

12 Salaun $E$, Jacquier $A$, Theron A, et al. Value of CMR in quantification of paravalvular aortic regurgitation after TAVI. Eur Heart J Cardiovasc Imaging 2016;17:41-50.

13 Frick M, Meyer CG, Kirschfink A, et al. Evaluation of aortic regurgitation after transcatheter aortic valve implantation: aortic root angiography in comparison to cardiac magnetic resonance. Eurolntervention 2016;11:1419-27.

14 Altiok E, Frick M, Meyer CG, et al. Comparison of two- and three-dimensional transthoracic echocardiography to cardiac magnetic resonance imaging for assessment of paravalvular regurgitation after transcatheter aortic valve implantation. Am J Cardiol 2014;113:1859-66.

15 Chaturvedi A, Hamilton-Craig C, Cawley PJ, et al. Quantitating aortic regurgitation by cardiovascular magnetic resonance: significant variations due to slice location and breath holding. Eur Radiol 2016;26:3180-9.

16 Gabriel RS, Renapurkar R, Bolen MA, et al. Comparison of severity of aortic regurgitation by cardiovascular magnetic resonance versus transthoracic echocardiography. Am J Cardiol 2011;108:1014-20.

17 Bertelsen L, Svendsen JH, Køber L, et al. Flow measurement at the aortic root - impact of location of through-plane phase contrast velocity mapping. J Cardiovasc Magn Reson 2016:18:55.

18 O'Brien KR, Cowan BR, Jain M, et al. MRI phase contrast velocity and flow errors in turbulent stenotic jets. J Magn Reson Imaging 2008;28:210-8.

19 Kerr AJ, O'Brien K, Gabriel R, et al. Underestimation of Aortic Flow by CMR in Aortic Stenosis-Implications for Aortic Valve Area Assessment. Heart, Lung and Circulation; 18:S57-S8.

20 Nayak KS, Nielsen JF, Bernstein MA, et al. Cardiovascular magnetic resonance phase contrast imaging. J Cardiovasc Magn Reson 2015;17:71.

21 Greil G, Geva T, Maier SE, et al. Effect of acquisition parameters on the accuracy of velocity encoded cine magnetic resonance imaging blood flow measurements. J Magn Reson Imaging 2002;15:47-54.

22 Traber J, Wurche L, Dieringer MA, et al. Real-time phase contrast magnetic resonance imaging for assessment of haemodynamics: from phantom to patients. Eur Radiol 2016:26:986-96.

23 Iwamoto Y, Inage A, Tomlinson G, et al. Direct measurement of aortic regurgitation with phase-contrast magnetic resonance is inaccurate: proposal of an alternative method of quantification. Pediatr Radiol 2014;44:1358-69.

24 Goffinet C, Kersten V, Pouleur AC, et al. Comprehensive assessment of the severity and mechanism of aortic regurgitation using multidetector CT and MR. Eur Radiol 2010;20:326-36.

25 Bolen MA, Popovic ZB, Rajiah P, et al. Cardiac MR assessment of aortic regurgitation: holodiastolic flow reversal in the descending aorta helps stratify severity. Radiology 2011;260:98-104.

26 Harris AW, Krieger EV, Kim M, et al. Cardiac Magnetic Resonance Imaging Versus Transthoracic Echocardiography for Prediction of Outcomes in Chronic Aortic or Mitral Regurgitation. Am J Cardiol 2017;119:1074-81.
27 Myerson SG, d'Arcy J, Mohiaddin R, et al. Aortic regurgitation quantification using cardiovascular magnetic resonance: association with clinical outcome. Circulation 2012;126:1452-60.

28 Ribeiro HB, Orwat S, Hayek SS, et al. Cardiovascular Magnetic Resonance to Evaluate Aortic Regurgitation After Transcatheter Aortic Valve Replacement. J Am Coll Cardiol 2016:68:577-85.

29 Gelfand EV, Hughes S, Hauser TH, et al. Severity of mitral and aortic regurgitation as assessed by cardiovascular magnetic resonance: optimizing correlation with Doppler echocardiography. J Cardiovasc Magn Reson 2006;8:503-7.

30 Bennett CJ, Maleszewski JJ, Araoz PA. CT and MR imaging of the aortic valve: radiologic-pathologic correlation. Radiographics 2012;32:1399-420.

31 Tamburino C, Capodanno D, Ramondo A, et al. Incidence and predictors of early and late mortality after transcatheter aortic valve implantation in 663 patients with severe aortic stenosis. Circulation 2011;123:299-308.

32 Hayashida K, Lefèvre T, Chevalier B, et al. Impact of post-procedural aortic regurgitation on mortality after transcatheter aortic valve implantation. JACC Cardiovasc Interv 2012;5:1247-56.

33 Mack MJ, Leon MB, Smith CR, et al. 5-year outcomes of transcatheter aortic valve replacement or surgical aortic valve replacement for high surgical risk patients with aortic stenosis (PARTNER 1): a randomised controlled trial. The Lancet 2015;385:2477-84.

34 Généreux P, Head SJ, Hahn R, et al. Paravalvular leak after transcatheter aortic valve replacement: the new Achilles' heel? A comprehensive review of the literature. J Am Coll Cardiol 2013;61:1125-36.

35 Kappetein AP, Head SJ, Généreux P, et al. Updated standardized endpoint definitions for transcatheter aortic valve implantation: the Valve Academic Research Consortium-2 consensus document (VARC-2). Eur J Cardiothorac Surg 2012;42:S45-S60.

36 Crouch G, Tully PJ, Bennetts J, et al. Quantitative assessment of paravalvular regurgitation following transcatheter aortic valve replacement. J Cardiovasc Magn Reson 2015;17:32

37 Ribeiro HB, Le Ven F, Larose $E$, et al. Cardiac magnetic resonance versus transthoracic echocardiography for the assessment and quantification of aortic regurgitation in patients undergoing transcatheter aortic valve implantation. Heart 2014;100:1924-32.

38 Orwat S, Diller GP, Kaleschke G, et al. Aortic regurgitation severity after transcatheter aortic valve implantation is underestimated by echocardiography compared with MRI. Heart 2014;100:1933-8.

39 Sherif MA, Abdel-Wahab M, Beurich HW, et al. Haemodynamic evaluation of aortic regurgitation after transcatheter aortic valve implantation using cardiovascular magnetic resonance. Eurolntervention 2011;7:57-63.

40 Schnell S, Entezari P, Mahadewia RJ, et al. Improved Semiautomated 4D Flow MR Analysis in the Aorta in Patients With Congenital Aortic Valve Anomalies Versus Tricuspid Aortic Valves. J Comput Assist Tomogr 2016;40:102-8.

41 Chelu RG, van den Bosch AE, van Kranenburg M, et al. Qualitative grading of aortic regurgitation: a pilot study comparing CMR 4D flow and echocardiography. Int J Cardiovasc Imaging 2016;32:301-7.

42 Hsiao A, Alley MT, Massaband P, et al. Improved cardiovascular flow quantification with time-resolved volumetric phase-contrast MRI. Pediatr Radiol 2011;41:711-20.

43 Wollmuth JR, Bree DR, Cupps BP, et al. Left ventricular wall stress in patients with severe aortic insufficiency with finite element analysis. Ann Thorac Surg 2006:82:840-6.

44 Murai S, Hamada S, Ueguchi T, et al. Aortic compliance in patients with aortic regurgitation: evaluation with magnetic resonance imaging. Radiat Med 2005:23:236-41. 